RAE-IC, Revista de la Asociación Española de Investigación de la Comunicación

vol. 8, núm. 15 (2021), 267-291

ISSN 2341-2690

Recibido el 30 de noviembre de 2020

DOI: https://doi.org/10.24137/raeic.8.15.12

Aceptado el 23 de diciembre de 2020

(c) (i) ()

\title{
Economía y financiación de medios en lenguas minoritarias celtas de Irlanda y Reino Unido: el modelo público como referencia
}

Economy and Funding in Celtic Minority Language Communities of the United Kingdom and Ireland: The Public Media Model as Paradigm

\author{
Zabaleta Urkiola, Iñaki \\ Universidad del País Vasco (UPV-EJU) \\ inaki.zabaleta@ehu.eus \\ Arriaga Azkarate, Tania \\ Universidad del País Vasco (UPV-EJU) \\ tania.arriaga@ehu.eus
}

Gorosarri González, María

Universidad del País Vasco (UPV-EJU)

maria.gorosarri@ehu.eus

Xamardo González, Nicolás

Universidad del País Vasco (UPV-EJU)

nxamardo@telefonica.net

Forma de citar este artículo:

Zabaleta Urkiola, I., Arriaga Azkarate, T., Gorosarri González, M. y Xamardo González, N. (2021). Economía y financiación de medios en lenguas minoritarias celtas de Irlanda y Reino Unido: el 
modelo público como referencia. RAE-IC, Revista de la Asociación Española de Investigación de la Comunicación, 8(15), 267-291. https://doi.org/10.24137/raeic.8.15.12

\section{Resumen:}

El artículo investiga los medios de comunicación monolingües en tres comunidades lingüísticas minoritarias celtas del Reino Unido e Irlanda, al final de la crisis económica iniciada en 2008 , desde la perspectiva del modelo de interés público, prevalente en los sistemas mediáticos en las lenguas minoritarias europeas. Las principales áreas de estudio son el volumen económico, el sistema de financiación y la evaluación cualitativa de editores y directores de medios en torno al efecto de la crisis económica y la importancia de la ayuda pública. Las tres lenguas minorizadas analizadas son el galés, el irlandés y el gaélico escocés. El diseño metodológico se basa en las técnicas de comparación cuantitativa sistemática entre comunidades y enfoque cualitativo. Entre los principales resultados y conclusiones se puede señalar que el volumen económico de los ingresos anuales de los tres sistemas de medios es superior a 200 millones de euros, de los que el $98 \%$ pertenece a medios públicos de radio y televisión, complementado por un reducido, aunque creciente sector de cibermedios y una prensa escrita en serio declive. El impacto de la crisis económica ha sido fuerte en las tres comunidades, pero la demanda de una mayor financiación pública ha sido más notoria entre los directores de medios de Irlanda y Escocia.

Palabras clave: lenguas minoritarias galesa irlandesa y gaélico escocesa, economía y financiación de los medios, ayudas públicas, volumen económico, densidad económica.

\section{Abstract:}

This article investigates the monolingual media in three Celtic minority language communities of the United Kingdom and Ireland, at the end of the economic crisis that began in 2008, from the perspective of the public interest media model, prevalent in the media systems of European minority language media. The main areas of study are the economic volume, the funding system and the qualitative evaluation of editors and 
media directors regarding the effect of the economic crisis and the importance of public aid. The three minority Celtic languages analyzed are Welsh, Irish and Scottish Gaelic. The methodological design is based on the techniques of systematic quantitative comparison between communities and a qualitative approach. Among the main results and conclusions, it can be noted that the economic volume of the annual income of the three media systems is greater than 200 million euros, of which $98 \%$ belongs to public radio and television, complemented with a very small, though growing, sector of cybermedia, and seriously declining print media. The impact of the economic crisis has been severe in the three communities, but the request for more public funding has been more evident in Ireland and Scotland.

Keywords: Welsh, Irish and Scottish Gaelic minority languages, media economy and funding, public media aid, economic volume, economic density.

\section{INTRODUCCIÓN}

La crisis económica que estalló en torno al 2008/2009 en EEUU y Europa y la rápida aceleración de la nueva era digital y tecnologías online han afectado severamente a la industria de los medios de comunicación y a sus modelos de negocio (Elliott, 2011; Franklin, 2016; Mylonas, 2012). Esta doble crisis ha sido visible y objeto de estudio en los medios generalistas, pero no existen datos fiables de lo que ha ocurrido en los medios informativos de comunidades lingüísticas minoritarias/minorizadasi.

A pesar de que los objetivos y funciones de los medios están principalmente relacionados con la salud democrática de la sociedad y el desarrollo de la comunidad, la empresa periodística, en tanto que negocio dedicado a producir contenidos e información, es un elemento crucial. Por ello, este trabajo investiga el peso económico y la estructura financiera de los medios de comunicación monolingües en tres comunidades lingüísticas celtas (galesa, irlandesa y gaélico escocesa), así como la evaluación proporcionada por sus editores en torno a las consecuencias provocadas por 
la crisis económica y la posible insuficiencia de la ayuda pública. El punto temporal de la investigación se sitúa en 2015/2016, inicio del finalii de la crisis económica.

El marco teórico, desde la perspectiva de la economía de la industria de los medios, se fundamenta principalmente en el modelo de interés público (Tunstall, 1991), en el que los medios de comunicación son responsables de su actividad ante la sociedad y las instituciones públicas, y el éxito se mide principalmente en función del interés público (Croteau y Hoynes, 2001).

Existe una notable cantidad de estudios que relacionan las lenguas minoritarias con diversos aspectos de la economía y la digitalización. Entre los temas investigados podemos mencionar el referido a los mercados, el comercio electrónico y la mercantilización (Cunliffe, Pearson y Richards, 2010; G. Hogan-Brun y O’Rourke, 2018; Pietikäinen, Kelly-Holmes y Rieder, 2019); la contribución de algunos proyectos de financiación de medios al idioma, como la televisión pública escocesa $B B C$ Alba (Chalmers, et al. 2012); la evaluación de la rentabilidad de las políticas referidas a las lenguas minoritarias (François Grin y Vaillancourt, 1999); el triángulo de la demanda, la oferta y el uso de los medios de comunicación en lenguas minoritarias (Broughton Micova, 2013); e incluso la economía de entornos multilingües (Grin, Sfreddo y Vaillancourt, 2010).

La unidad de análisis de este estudio es la comunidad lingüística en su totalidad, y no una región política o administrativa en que pueda estar dividida la comunidad de un idioma, como ocurre en el caso de Irlanda. De este modo, se comparan los tres sistemas de medios de modo directo y sistemático con el objeto de conocer la realidad global de los medios en lenguas celtas del Reino Unido e Irlanda.

Los conceptos de interés público y esfera pública (McQuail, 2000/1983; Rogers, 1995) son la base sobre la que los gobiernos impulsan políticas de regulación y apoyo financiero a los medios de comunicación en lenguas mayoritarias $y$, asimismo, a los medios de comunicación en lenguas minorizadas; políticas que, a menudo, son "determinantes en la actuación económica de los mercados de los medios" (Doyle, 2002, p. 161). 
En el caso de los sistemas de medios en lenguas minorizadas, la importancia de la financiación pública se presenta incluso más relevante debido a la debilidad económica de estas empresas. De hecho, la gran mayoría de los medios de comunicación en lenguas minoritarias, tanto de propiedad social como privada, de distintos países (España, Reino Unido y los Países Bajos, por ejemplo) obtienen una importante financiación pública para reequilibrar sus presupuestos (Zabaleta y Xamardo, 2020), debido a que las fuentes de ingresos ordinarios a menudo son insuficientes.

Con respecto a las tres comunidades lingüísticas analizadas, los rasgos fundamentales de su lengua y sus sistemas de medios son los siguientes: el número aproximado de hablantes ${ }^{\mathrm{iii}}$ es de 0,6 millones (21\% de la población) en Gales; 0,8 millones (12\%) en Irlanda, incluyendo la República de Irlanda e Irlanda del Norte; y 60.000 hablantes en Escocia (1\%). Por otro lado, la estructura de medios monolingües (Zabaleta et al., 2019) se puede resumir en los siguientes datos (Tabla 1):

- El sistema mediático en lengua galesa consta de un considerable número de organizaciones periodísticas, 84 concretamente. Los medios más importantes, que ejercen de columna vertebral del sistema, son el canal público de televisión S4C, la radio pública $B B C$ Radio Cymru, la revista de propiedad privada semanal Golwg ${ }^{\text {iv }}$, y los medios digitales Golwg 360 y BBC Cymru Fyw (considerados como plataformas diferenciadas de sus correspondientes medios tradicionales). También es relevante subrayar el valor de 59 revistas locales, de propiedad social y publicación mensual, denominadas Papurau Bro.

- El sistema de medios en lengua irlandesa, incluyendo el norte y el sur de la comunidad, es más débil que el galés. Cuenta con 12 medios únicamente, entre los que sobresalen la televisión pública Telifis na Gaelige TG4, la estación de radio pública Raidió na Gaeltachta y tres medios nativos digitales Nós, Tuairisc.ie y Meon Eile. Hay muy pocos medios de difusión local y carecen de un diario o un semanario de prensa impresa. De hecho, el sector de la prensa escrita monolingüe en irlandés es muy débil.

- En Escocia solo hay dos medios de comunicación en lengua gaélico escocesa, ambos de propiedad pública y difusión general: la radio Radio nan Gàidheal, 
servicio ofrecido por la BBC de Escocia, y el canal de televisión $B B C$ Alba, propiedad de la BBC y MG Alba. La comunidad lingüística en cuestión carece de prensa escrita.

\section{REVISIÓN DE LA LITERATURA}

\subsection{MODELOS, FINANCIACIÓN Y CRISIS ECONÓMICA}

La investigación académica ha introducido dos modelos genéricos para enmarcar la industria periodística: el modelo de mercado y el modelo de interés público (Croteau y Hoynes, 2001; Tunstall, 1991).

En el modelo de mercado, los medios de comunicación se conceptualizan como empresas que venden contenidos y servicios, con el propósito clave de generar ganancias económicas, dejando el progreso democrático de los ciudadanos y la sociedad en un nivel más secundario, aunque importante. Las audiencias son contempladas predominantemente como consumidores, y se considera que la regulación interfiere en la actividad del mercado.

El modelo de esfera público, en cambio, concibe los medios de comunicación como un recurso social al servicio de la comunidad, cuyo objetivo principal es "promover la ciudadanía activa". La regulación se percibe como una herramienta útil para proteger el interés público. La innovación tiene como objetivo fomentar la participación, y la diversidad se define a lo largo de varias vías (contenido, representación social, perspectivas ideológicas, presencia minoritaria, etc.). Por tanto, los medios de comunicación son responsables ante la sociedad y las instituciones públicas y el éxito se mide principalmente en función del interés público (Croteau y Hoynes, 2001, p. 37).

Se puede afirmar que la mayor parte de los medios en lenguas minoritarias, en Europa y en otras partes del mundo, está más cerca del modelo de interés público, aunque el modelo de mercado también tiene una pequeña participación en algunas comunidades lingüísticas. 
Pero incluso en el modelo de interés público la situación y evolución de la economía de los medios es fundamental para su subsistencia y su función social. En este sentido, se sabe que la financiación de los medios se ha visto perturbada por una recesión económica general que comenzó alrededor de 2008/2009 (Dragomir, 2018; Elliott, 2011; LEAP/Europe 2020, 2011) y por una crisis específica de los medios, desencadenada por un proceso de digitalización acelerado que ha producido profundos cambios en las áreas de la tecnología, las regulaciones, los modelos de negocio y, sin duda, en las demandas sociales (Benson, 2019; Levy y Nielsen, 2010; Nobre Correia, 2010). La globalización de la industria y la canibalización de contenidos por parte de actores globales, así como la nueva división del mercado publicitario, son otros factores relevantes de cambio (Lowe y Berg, 2013).

Como consecuencia de esta crisis combinada, muchos medios han reducido su presupuesto y su plantilla de empleados (Kevin, Pellicanò y Schneeberger, 2013; Westcott, 2015). De hecho, desde el comienzo del milenio, los servicios públicos de radiodifusión han sufrido reducciones significativas de fondos en muchos países (Hanley, 2003; Westcott, 2015). Pero, teniendo en cuenta que en Europa existen diferentes modelos de financiación de los servicios públicos de radiodifusión, el tamaño y la riqueza de los países se convierten en factores discriminatorios. En consecuencia, el modelo de los países ricos no es apropiado para los países más pequeños o económicamente más débiles (Lowe y Berg, 2013).

Los medios de comunicación en lenguas minoritarias no han escapado a esos y otros efectos negativos. Ha habido cierres de medios de comunicación debido a la reducción o cancelación de ayudas públicas. Por ejemplo, en Escocia, An Gaidheal Ur, el único periódico impreso monolingüe en gaélico escocés dejó de publicarse en 2009, debido a una reducción significativa de las subvenciones públicas. Razones similares se escondieron detrás del cierre en 2008 del único diario en irlandés Lá y del semanario Gaelscéal en 2013.

\subsection{LENGUAS MINORITARIAS: LEGISLACIÓN Y ESTUDIOS}


En Europa, los dos marcos legales para apoyar las lenguas regionales y minoritarias, y ayudar financieramente a sus medios de comunicación, son la Carta Europea de las Lenguas Minoritarias o Regionales ${ }^{v}$ y el Convenio Marco para la Protección de las Minorías Nacionales, ambos aprobados por el Consejo Europeo.

La Carta, forma abreviada como se la conoce, clasifica las lenguas en cinco grupos: lenguas nacionales de pequeños Estado-nación que tienen un uso y desarrollo minoritario y se encuentras amenazadas (como en el caso del idioma irlandés); lenguas de comunidades que existen en un único Estado-nación (como el galés y el gaélico escocés); lenguas de comunidades que residen en dos o más Estados-nación (vasco y catalán); lenguas que son minoría en un Estado-nación pero son la lengua principal en otros países (por ejemplo, el hindi en Gran Bretaña o el turco en Alemania), y las lenguas no territoriales como el romaní. Las lenguas celtas que investigamos en este estudio forman parte de las categorías uno y dos.

La Carta entró en vigor en 1998 y ha sido ratificada por 27 países; 18 estados no la han ratificado; y 14 aún no la han firmado ni ratificado, entre los cuales se encuentra Irlanda, debido a que el irlandés es un idioma oficial del estado y, por tanto, no entra dentro del marco definitorio de la Carta Europea.

Existen numerosos estudios académicos que profundizan en distintos aspectos de las lenguas étnicas, autóctonas, regionales y/o minorizadas, pero toman como referencia comunidades individuales. Otros recurren a una agregación de casos particulares, con lo que se obtiene una comparación indirecta entre comunidades. Así, cabe mencionar las actas de los diversos congresos internacionales sobre lenguas minoritarias (Gorter, Hoekstra, Jansma, y Ytsma, 1989); la publicación de Riggins (1992, p. 1), donde se presentan cinco modelos (integracionista, económico, divisivo, preventivo y proselitista) de estrategias del Estado con respecto a los medios de comunicación de comunidades lingüísticas minoritarias; el volumen de Hogan-Brun y Wolff (2003) sobre lenguas autóctonas y diaspóricas en Europa; el libro de Kirk Baoill (2003) sobre las lenguas minoritarias de Irlanda y Escocia; el trabajo de Cormack y Hourigan (2007), que recopila trabajos europeos y reflexiona sobre ellos; el volumen editado por Jones and Uribe- 
Jongbloed (2012) sobre la confluencia de las industrias creativas; y el concepto de completitud institucional, funcional y de géneros de contenidos (Moring, 2007).

En un tercer grupo se encuadran los estudios de comparación sistemática intercomunitaria como, por ejemplo, el referido al desarrollo de las plataformas online y redes sociales en lenguas minoritarias europeas (Ferré-Pavia, Zabaleta, Gutierrez, Fernandez-Astobiza, y Xamardo, 2018; Zabaleta et al., 2013); los roles y funciones de los periodistas (Zabaleta et al., 2010); y la estructura de los sistemas de los medios de comunicación (Zabaleta et al. 2014; Zabaleta et al. 2019).

\section{OBJETIVOS E HIPÓTESIS}

10 objetivo: estudio del volumen económico de los sistemas de medios de comunicación en las comunidades lingüísticas irlandesa, galesa y gaélico escocesa, en función de las variables de tipo de medio, propiedad y difusión en el 2015.

2o objetivo: cálculo de la densidad económica o peso económico relativo en función del número de hablantes en la lengua minoritaria y, por otro, de la población en general.

3 objetivo: análisis de la estructura de financiación de los cuatro tipos de medios (prensa, radio, TV y cibermedios o medios digitales nativos) en las tres comunidades analizadas.

40 objetivo: investigación de la percepción que los editores de los medios y los directores financieros de las tres comunidades tienen sobre el impacto de la crisis económica y la suficiencia o insuficiencia de la financiación y ayuda públicas.

\section{METOdOLOGÍA}

Se han implementado dos métodos diferentes, ambos adecuados para los objetivos de la investigación: cuantitativo para el estudio del volumen o peso económico, de la densidad económica de los medios, y de la estructura financiera; y cualitativo para el informe de la percepción que los editores tienen sobre el efecto de la crisis económica, así como sobre la suficiencia o insuficiencia de las subvenciones públicas. 
La definición operativa del volumen o peso económico (Zabaleta et al., 2020), también comúnmente conocido como volumen de negocio, se establece como el ingreso total anual de un medio de comunicación a través de sus diversas fuentes de financiación (publicidad, ventas, subscripciones, financiación pública, y otras). Por otro lado, el concepto de densidad económica es definido como la relación entre el volumen económico y la población de hablantes de una comunidad lingüística o la población total. Operativamente se representa como la cantidad de euros del volumen económico que se invierte por, o corresponde a, cada hablante o cada persona de la sociedad analizada. Este concepto es importante para evaluar el grado relativo de inversión económica que bien una sociedad o bien una comunidad lingüística aporta a su sistema de medios, lo que permite realizar comparaciones proporcionales intercomunitarias. En cuanto a la unidad de moneda, puesto que en las comunidades galesa, escocesa y norirlandesa los ingresos de los medios son en libras esterlinas, estas cifras se han convertido a euros.

En el caso de la estructura financiera, los resultados, correspondientes a 2015, responden a cuatro categorías de ingresos: financiación pública, otorgada por distintas instituciones y por diferentes conceptos (impuesto por licencia, subvenciones); publicidad, incluida la comercial y la institucional; ventas y subscripciones, principalmente en el caso de la prensa impresa; y otro tipo de ingresos (colectas, sorteos, etc.).

La recopilación de los datos se ha llevado a cabo utilizando técnicas diferentes: entrevistas en directo con los directores de los medios y los directores financieros en los propios medios; cuestionarios online; análisis de los balances contables y los informes financieros anuales; y viajes a las comunidades y los medios de difusión para identificar el día a día, la realidad tras los datos económicos ${ }^{\mathrm{vi}}$.

En los sistemas de medios en lengua irlandesa y gaélico escocesa, el volumen económico, la densidad y la estructura de la financiación se han calculado empleando el censo de medios, esto es, obteniendo datos de todas las organizaciones periodísticas. En el sistema de medios en lengua galesa, también se empleó el censo en el caso de los 
medios de difusión general (distribución a toda la comunidad) para calcular el volumen y la densidad económicos. Sin embargo, en los medios locales se diseñó una muestra estratificada y con selección aleatoria de elementos y se lograron datos de 34 medios, de los 59 existentes.

Se estima que el nivel de error es inferior al $3 \%$, ya que alrededor del $90 \%$ del volumen económico se ha obtenido empleando datos de los censos de medios.

El cuestionario dirigido a los directores y editores de los medios se ha dividido en dos secciones. En el apartado correspondiente a las preguntas cerradas se han incluido cuestiones sobre las características del medio (tipo, propiedad, difusión), el volumen económico y estructura e ingresos de financiación. La sección de preguntas abiertas ha permitido que respondieran ad libitum sobre el efecto de la crisis económica y expresaran su opinión en torno a la financiación y a la suficiencia o insuficiencia de las ayudas públicas vii.

Las empresas periodísticas analizadas en este trabajo son medios monolingües, definidos operativamente como aquellos tipos de medios (prensa escrita, radio, TV y cibermedios o nativos digitales) en los que al menos el $70 \%$ del contenido está en la lengua minoritaria, su frecuencia de publicación es al menos semestral, presentan contenido general o especializado y una difusión o topos de carácter general o local. Su propiedad puede ser pública, privada o social, esto es, perteneciente a organizaciones sin ánimo de lucro.

\section{RESULTADOS}

\subsection{VOLUMEN ECONÓMICO Y DENSIDAD}

El volumen económico de los tres sistemas de medios era de 213,1 millones de euros en 2015.

Los medios en lengua galesa alcanzan un ingreso total anual de 140,5 millones, los de lengua irlandesa bastante menos de la mitad (50,0 millones de euros), y los de lengua gaélico escocesa 20,6 millones de euros. Por tanto, en las tres comunidades minoritarias 
celtas analizadas, cerca de dos tercios (65,9\%) del volumen económico está en Gales, menos de un cuarto $(23,5 \%)$ en Irlanda, y solo el $10,6 \%$ en Escocia.

En relación al tipo de medios, el 83,9\% del total de los ingresos anuales pertenece a la televisión, el 13,0\% a la radio, el 1,1\% a la prensa escrita, y el 2,0\% a los cibermedios.

Cabe resaltar que el volumen económico de los medios nativos digitales (4,4 millones de EUR) es superior al de la prensa escrita (2,3 millones de EUR). Con respecto a los cibermedios, en Irlanda existen tres (Nós, Tuairisc.ie y Meon Eile) y en Gales otros tres (BBC Cymru Fyw de la BBC Wales viii; Pobl Caerdydd, de carácter local cuyo nombre significa "People of Cardiff"; y Y Papur Gwyrdd, dedicado al medioambiente).

Tabla 1. Volumen económico por tipo de medios (2015)

\begin{tabular}{|c|c|c|c|c|c|c|c|c|}
\hline $\mathbf{( 1 )}$ & $\begin{array}{c}\text { Vol. } \\
\text { Econ. }\end{array}$ & $\begin{array}{c}\text { Porcentaje } \\
\text { vertical }\end{array}$ & & Prensa & Radio & TV & Ciber & Total \\
\cline { 1 - 2 } \cline { 5 - 8 } Galesa & 140,5 & $65,9 \%$ & $1,5 \%$ & $8,5 \%$ & $87,4 \%$ & $2,6 \%$ & $100 \%$ \\
\hline Irlandesa & 50 & $23,5 \%$ & $0,5 \%$ & $22,2 \%$ & $75,9 \%$ & $1,4 \%$ & $100 \%$ \\
\hline G. escocesa & 22,6 & $10,6 \%$ & $0,0 \%$ & $20,2 \%$ & $79,8 \%$ & $0,0 \%$ & $100 \%$ \\
\hline Total & 213,1 & - & 2,3 & 27,6 & 178,7 & 4,4 & 213,1 \\
\hline Total $\%$ & - & $100 \%$ & & $1,1 \%$ & $13 \%$ & $89,3 \%$ & $2 \%$ & $100 \%$ \\
\hline
\end{tabular}

Fuente: elaboración propia. N: 76 medios monolingües. Notas: (1) Las cifras que no corresponden a porcentajes representan millones de euros.

En lo que respecta a la propiedad de los medios (Tabla 3), el 98,3\% del total del volumen económico de los medios monolingües de las tres comunidades celtas pertenece a medios de propiedad pública, el 0,9\% a medios privados y el 0,8\% a medios de propiedad social.

Tabla 2. Volumen económico por propiedad de medios (2015)

\begin{tabular}{|c|c|c|c|c|}
\hline & Radio & TV & Ciber & Total \\
\hline Galesa & $98,5 \%$ & $0,8 \%$ & $0,7 \%$ & $100 \%$ \\
\hline Irlandesa & $96,9 \%$ & $1,7 \%$ & $1,4 \%$ & $100 \%$ \\
\hline G. escocesa & $100 \%$ & $0 \%$ & $0 \%$ & $100 \%$ \\
\hline Total & $98,3 \%$ & $0,9 \%$ & $0,8 \%$ & $100 \%$ \\
\hline
\end{tabular}

Fuente: elaboración propia. N: 76 medios monolingües.

Por último, con respecto a la variable de la difusión o topos, el 99,4\% del volumen económico está en manos de los medios de difusión general, esto es, de los que difunden sus contenidos a toda la comunidad lingüística o política, y sólo el $0,6 \%$ pertenece a los medios de difusión local. Más en concreto, en la comunidad lingüística 
gaélico escocesa no se encuentra ningún medio local ni, por tanto, ningún peso económico; y en las comunidades lingüísticas galesa e irlandesa su valor es mínimo.

\subsection{DENSIDAD ECONÓMICA}

Más allá del concepto de volumen económico, también es importante analizar la densidad económica - esto es, cuánto dinero se invierte por cada habitante y/o hablante- con el objeto de evaluar su peso relativo y el grado de inversión económica en la sociedad en general y en la comunidad de hablantes de la lengua minoritaria en particular.

En 2015, la densidad económica total (Tabla 3) del conjunto de las tres comunidades analizadas era de $14 €$ por habitante y $152 €$ por hablante, valor que en $2019 / 2020$ permanece casi invariado.

Tabla 3. Densidad económica (2015)

\begin{tabular}{|c|c|c|c|c|c|}
\hline & $\begin{array}{c}\text { Población } \\
\text { (millones) }\end{array}$ & $\begin{array}{c}\text { Habitantes } \\
\text { (millones) }\end{array}$ & $\begin{array}{c}\text { Vol. Econ. } \\
\text { (millones } \boldsymbol{\text { (m) }}\end{array}$ & $\begin{array}{c}\text { Dens. Econ. } \\
\text { Por habitante } \\
(\boldsymbol{\epsilon})\end{array}$ & $\begin{array}{c}\text { Dens. Econ. } \\
\text { Por hablante } \\
(\boldsymbol{(})\end{array}$ \\
\hline Galesa & 2,7 & 0,6 & 140,5 & 52 & 246 \\
\hline Irlandesa & 6,6 & 0,8 & 50 & 8 & 65 \\
\hline G. escocesa & 5,4 & 0,1 & 22,6 & 4 & 376 \\
\hline Total & 14,7 & 1,4 & 213,1 & 14 & 152 \\
\hline
\end{tabular}

Fuente: elaboración propia.

Si se realiza una comparación intercomunitaria, en las comunidades lingüísticas galesa $y$, sobre todo escocesa, la densidad económica es alta, notablemente por encima de los $200 €$ por cada hablante (246 euros en la galesa y $376 €$ en la escocesa). El resultado claramente negativo se encuentra en la comunidad lingüística irlandesa, donde la densidad económica es inferior a los cien euros (65 euros concretamente).

Pero si consideramos la densidad económica desde la variable de la población, observamos que en Irlanda y Escocia los valores son inferiores a 10 euros (8 y 4 euros respectivamente) y en Gales asciende a la importante cifra de 52 euros.

\subsection{SISTEMA DE FINANCIACIÓN}


En los tres sistemas de medios, casi toda la financiación (Tabla 4) proviene de los ingresos proporcionados por las instituciones públicas (96,0\%); el 3,4\%, de la publicidad; el $0,3 \%$, de ventas y suscripciones; y el $0,3 \%$, de recursos varios como venta de programas y/o derechos, actividades sociales para la recaudación de fondos, etc.

Tabla 4. Sistema de financiación (2015)

\begin{tabular}{|c|c|c|c|c|c|c|}
\hline & $\begin{array}{c}\text { Vol. Econ. } \\
\text { (millones de } € \text { ) }\end{array}$ & $\begin{array}{c}\text { Finan. } \\
\text { Pública }\end{array}$ & Publicidad & $\begin{array}{c}\text { Ventas y } \\
\text { subs. }\end{array}$ & Otros & Total \\
\hline Galesa & 2140,5 & $96,6 \%$ & $2,8 \%$ & $0,4 \%$ & $0,2 \%$ & $100 \%$ \\
\hline Irlandesa & 50 & $93,3 \%$ & $6,3 \%$ & $0,1 \%$ & $0,3 \%$ & $100 \%$ \\
\hline G. escocesa & $22,6 \%$ & $99,2 \%$ & $0 \%$ & $0 \%$ & $0,8 \&$ & $100 \%$ \\
\hline Total & 213,1 & $96 \%$ & $3,4 \%$ & $0,3 \%$ & $0,3 \%$ & $100 \%$ \\
\hline
\end{tabular}

Fuente: elaboración propia. Nota: (1) N = 46 medios. Datos de estructura financiera obtenidos de todos los medios en lenguas irlandesa y gaélico escocesa y de 34 medios en lengua galesa, incluyendo todos los medios principales y una muestra adecuada de Papurau Bro.

Con respecto a la distribución de la financiación en función de la propiedad de los medios (Tabla 5), de nuevo es la financiación pública la fuente de ingresos mayoritaria, aunque hay matices importantes en el análisis de esta variable. Considerando el total de las tres comunidades, los resultados son los siguientes:

Financiación pública: el 96,7\% de la financiación en los medios públicos proviene de las instituciones públicas, en el caso de los medios privados este tipo de ingreso se reduce al $52,1 \%$ y en los medios sin ánimo de lucro al $65,8 \%$.

Publicidad: el ingreso por publicidad alcanza el $17,4 \%$ en los medios privados, el 3,2\% en los públicos y el $5,2 \%$ en los medios de propiedad social.

Ventas y subscripciones: este tipo de ingreso representa el $16,6 \%$ del total en los medios privados y el $20,6 \%$ en los medios sin ánimo de lucro. Evidentemente, los medios públicos, pertenecientes a la radiotelevisión, no existe este tipo de financiación. Tabla 5. Sistema de financiación según propiedad medios (2015)

\begin{tabular}{|c|c|c|c|c|c|c|c|c|c|c|c|c|}
\hline (1) (2) & \multicolumn{2}{|c|}{ Financiación pública } & \multicolumn{3}{|c|}{ Publicidad } & \multicolumn{2}{c|}{$\begin{array}{c}\text { Ventas y } \\
\text { subscripciones }\end{array}$} & \multicolumn{3}{c|}{ Otros } \\
\hline & Priv. & Pub. & Soc. & Priv. & Pub. & Soc. & Priv. & Pub. & Soc. & Priv. & Pub. & Soc. \\
\hline Galesa & $18.2 \%$ & $97.4 \%$ & $48.4 \%$ & $29.1 \%$ & $2.6 \%$ & $6.9 \%$ & $34.1 \%$ & $0.0 \%$ & $36.5 \%$ & $18.6 \%$ & $0.0 \%$ & $8.2 \%$ \\
\hline Irlandesa & $79.4 \%$ & $93.7 \%$ & $82.2 \%$ & $8.1 \%$ & $6.3 \%$ & $3.7 \%$ & $2.5 \%$ & $0.0 \%$ & $5.7 \%$ & $10.1 \%$ & $0.0 \%$ & $8.4 \%$ \\
\hline
\end{tabular}




\begin{tabular}{|c|c|c|c|c|c|c|c|c|c|c|c|c|}
\hline $\begin{array}{c}\text { G. } \\
\text { escocesa }\end{array}$ & - & $99.2 \%$ & - & - & $0.0 \%$ & - & - & $0.0 \%$ & - & - & $0.8 \%$ & \\
\hline Total & $52.1 \%$ & $96.7 \%$ & $65.8 \%$ & $17.4 \%$ & $3.2 \%$ & $5.2 \%$ & $16.6 \%$ & $0.0 \%$ & $20.6 \%$ & $13.9 \%$ & $0.1 \%$ & $8.3 \%$ \\
\hline
\end{tabular}

Fuente: elaboración propia. Nota: (1) N = 46 medios. Nota: (1) Las casillas vacías indican la inexistencia de entradas en esa categoría de propiedad.; (2) Priv.: Privado;

Pub.: Público; Soc.: propiedad social.

Otros: los ingresos obtenidos por otras actividades llegan al $13,9 \%$ en los medios privados, al 8,3\% en los sociales, y solo el $0,1 \%$ en los públicos.

La comparación entre las tres comunidades muestra unas diferencias relevantes. En los dos únicos medios existentes en lengua gaélico escocesa, ambos públicos, prácticamente toda la financiación es también pública $(99,2 \%)$, lógicamente. En cambio, la financiación pública presenta importantes diferencias en los medios privados galeses e irlandeses. En Gales, solo el 18,2\% de la financiación es pública en los medios privados, mientras que en Irlanda ese porcentaje sube al 79,4\%. Por otro lado, en los medios de propiedad social de Gales los fondos públicos anuales representan el $48,4 \%$ de la financiación anual y en Irlanda el $82,2 \%$. Por tanto, se puede concluir que las instituciones públicas de Irlanda financian con mayor proporción a los medios privados y sociales que las de Gales.

En el resto de las fuentes de financiación (publicidad, ventas y otros), las diferencias porcentuales, aunque aparentemente importantes, no tienen relevancia estadística porque las cantidades en euros son reducidas.

\subsection{IMPACTO Y EVALUACIÓN}

El análisis cuantitativo ofrecido se debe complementar, a su vez, con una visión cualitativa y un contexto evaluador de la situación derivada de la crisis económica y de la suficiencia o insuficiencia de las ayudas públicas.

Sobre el efecto de la crisis económica en la financiación de los medios, de los 35 directores/editores cuestionados en las tres comunidades lingüísticas, cerca de dos tercios (64\%) ha confirmado que les ha afectado negativamente, pero una cuarta parte (27\%) ha opinado lo contrario; el resto no se ha definido al respecto. 
Teniendo en cuenta la variable del tipo de medio, los gestores de los medios de prensa escrita y radiotelevisión son los que más han señalado el perjuicio de la crisis. Si a dicha variable le añadimos el de la propiedad de los medios, se observa que prácticamente el $100 \%$ de los gestores de medios públicos de las tres comunidades opina que ha sufrido recortes económicos. En la comunidad galesa, incluso los editores de los medios privados coinciden en esta conclusión. Con respecto a los medios de propiedad social, la mitad de los editores galeses de los Papurau Bro apuntan que la crisis económica les ha afectado, hecho en el que coinciden el $75 \%$ de los directores de medios sin ánimo de lucro irlandeses.

En torno a la suficiencia o insuficiencia de la financiación y las ayudas públicas, dos tercios de los directores de los medios en lengua irlandesa han expresado que son insuficientes y que es necesario su incremento. Por otro lado, los gestores de medios en lengua galesa aparecen divididos, ya que la mitad considera que son aceptables y la otra mitad lo contrario. Siguiendo con Gales, conviene señalar que un 7,7\% de sus medios en la lengua autóctona no solicita ayudas públicas puesto que desean mantener una independencia editorial y financiera. Por último, un editor del canal de televisión escocés $B B C$ Alba también ha subrayado la escasez de la financiación pública, a pesar del incremento de la audiencia.

\section{CONCLUSIONES}

El volumen económico anual de los sistemas de medios en lengua galesa, irlandesa y gaélico escocesa alcanzó la importante cifra de 213,1 millones de euros en 2015, cantidad que en 2019/2020 es similar, dentro de un margen de incremento en torno al $5 \%$, puesto que el balance anual de las tres radiotelevisiones públicas no ha variado significativamente (BBC, 2020; MG Alba, 2020; S4C, 2020). Pero las diferencias intercomunitarias son muy importantes, ya que alrededor de dos tercios del peso económico está en Gales, un cuarto en Irlanda (norte y sur), y solo un 10,6\% en Escocia. Por tanto, se puede argumentar que, desde la perspectiva económica, hay tres niveles diferenciados de desarrollo mediático. 
Debido a la propia naturaleza de los medios y a la inversión que se requiere para la producción de contenidos, la televisión absorbe cerca de ocho euros y medio de cada diez, la radio algo más de un euro, y el medio euro restante se reparte entre los cibermedios y la prensa escrita, aunque con el importante matiz de que los medios digitales superan a la prensa escrita con claridad. Precisamente, el sector digital, dentro de su limitado volumen económico de algo más de cuatro millones de euros, está teniendo un cierto desarrollo en Irlanda y Gales, mientras que la prensa escrita ha sido y es muy débil; e inexistente en Escocia.

Considerando la variable de la propiedad, algo más del $98 \%$ del volumen económico total pertenece a medios públicos y solo un escaso $2 \%$ a los medios de propiedad privada y social, ambos con valores similares.

La tercera variable, la de la difusión, también revela que el 99\% de la economía de los medios de las tres comunidades lingüísticas pertenece a los medios de difusión general y menos del $1 \%$ a los medios locales. Este resultado significa que los tres sistemas de medios están dirigidos a toda la comunidad lingüística o política y que el peso económico de los medios locales es muy reducido, aunque se debe reconocer la importancia social de los Papurau Bro galeses.

Por tanto, desde el punto de vista de la economía, los sistemas de medios de las tres comunidades lingüísticas se encuadran en el modelo de interés público, ya que son sistemas centrados en la radiotelevisión pública de difusión general a toda la comunidad, con un sector de cibermedios básico, parcialmente público, y una prensa escrita en serio declive.

Los hallazgos previos se refieren a los sistemas de medios y las comunidades tomadas como unidades, sin tener en cuenta el tamaño de la población general y de la cantidad de hablantes del idioma minoritario. Por tanto, para poder hacer comparaciones proporcionales es necesario recurrir al concepto de densidad económica desde dos variantes: por habitante y por hablante. 
En las comunidades lingüísticas galesa y gaélico escocesa, la densidad económica por hablante es alta, ya que la sociedad invierte en sus medios de lengua propia bastante más de doscientos euros por cada hablante ( $246 €$ y $376 €$ respectivamente). En cambio, en Irlanda, incluyendo la República de Irlanda e Irlanda del Norte, la inversión en medios propios por cada hablante de irlandés es de 65 euros, lo que significa que es un valor casi cuatro veces ( 3,8 veces inferior) menor que el galés y seis veces más reducido que el gaélico escocés ( 5,8 veces inferior).

Si empleamos el concepto de densidad económica por habitante, no solo los valores cambian significativamente, como cabe esperar, sino que también cambia el ranking de las comunidades. Así, en Irlanda y Escocia, los recursos económicos empleados en sus medios de lengua propia son inferiores a diez euros por habitante, mientras que en Gales es casi cinco veces superior, alcanzando 52 euros. El razonamiento que se puede hacer conduce a la conclusión de que, en Gales, para la población total existente, el esfuerzo inversor realizado en los medios de su lengua propia es importante, mientras que en Irlanda y Escocia es notablemente más reducido.

¿Pero son estos valores de densidad adecuados? Evidentemente, no existe un método directo de evaluación, por lo que se ha recurrido a un método indirecto, comparando las densidades económicas de los tres sistemas de medios con las densidades económicas de las corporaciones públicas de radiodifusión europeas.

En 2015, la densidad económica por habitante de las corporaciones públicas de radiotelevisión de la UE fue de 67 euros (Campos Freire, 2017); en el Reino Unido, la densidad de la BBC, 89 euros; y en Irlanda, la de la corporación irlandesa RTÉ, 90 eurosix.

Contrastando dichos valores con los de las tres comunidades, solo Gales, con $52 €$ por habitante, se acerca al índice europeo; aunque a distancia de los índices de la BBC y la RTÉ. En cambio, Irlanda y Escocia dedican menos de diez euros por habitante a sus medios de lengua autóctona, que predominantemente es radiotelevisión.

Pero si la comparación se hace con la densidad económica por hablante, los valores de Gales y Escocia están por encima de los doscientos euros, esto es, muy por encima de la 
densidad europea. Lamentablemente, Irlanda presenta un valor inferior incluso en esta vertiente de la densidad económica, lo que significa que los medios en lengua irlandesa cuentan con un volumen económico significativamente reducido en comparación con su comunidad de hablantes en irlandés.

Los resultados anteriores determinan una estructura de financiación similar en las tres comunidades, ya que nueve euros y medio corresponden a ingresos públicos, y el resto a publicidad, ventas y subscripciones, y otro tipo de ingresos.

Al dirigir la mirada al efecto de la crisis y la suficiencia o insuficiencia de las ayudas públicas, casi el cien por cien de los directores de medios públicos y la mayoría de los gestores de medios privados y sociales en las tres comunidades opinan que ha habido una reducción significativa en el volumen económico, con recortes a partir del inicio de la crisis de 2008. En relación a la insuficiencia de las ayudas públicas, la queja más importante se ha recibido en la comunidad lingüística irlandesa y gaélico escocesa, mientras que en Gales la percepción está dividida, debido en parte a la propia realidad mediática y a que algunos medios, para mantener su independencia editorial, no piden ayudas.

En definitiva, las implicaciones de este estudio permiten consolidar la conclusión de que los tres sistemas de medios pertenecen inequívocamente al modelo de interés público, tanto en el volumen económico como en la estructura de la financiación. No obstante, también se constata que las instituciones de las tres comunidades y sociedades pueden encontrarse ante la disyuntiva social y política sobre cuál debe ser la densidad económica de referencia para sus políticas de financiación, esto es, la densidad económica por hablante o por habitante. Una reflexión ponderada parece sugerir que debe ser una combinación de ambas, toda vez que la audiencia de los medios está abierta a toda la sociedad y, sobre todo, a que los medios en lenguas minoritarias son patrimonio de toda la comunidad. 


\section{RECONOCIMIENTOS}

Esta investigación ha sido financiada por la Universidad del País Vasco (UPV-EHU) y evaluada positivamente por la Comisión Nacional Evaluadora de la Actividad Investigadora (CNEAI) de España. Los autores agradecen a los directores y editores de los medios investigados su colaboración, al igual que a los representantes de las instituciones públicas irlandesas y galesas entrevistados para esta investigación.

\section{REFERENCIAS BIBLIOGRÁFICAS}

BBC. (2020). BBC Group Annual Report and Accounts 2019/20. bbc.co.uk. Recuperado de http://downloads.bbc.co.uk/aboutthebbc/reports/annualreport/2019-20.pdf

Benson, R. (2019). Paywalls and public knowledge: How can journalism provide quality news for everyone? Journalism, 20(1), 146-149.

https://doi.org/10.1177\%2F1464884918806733

Broughton Micova, S. (2013). Rights vs. reality: minority language broadcasting in South East Europe. Journal on Ethnopolitics and Minority Issues in Europe, 12(4), 54-79.

Campos Freire, F. (2017). Situación actual y tendencias de la radiotelevisión pública en Europa. Recuperado de www.researchgate.net/publication/318792202_Situacion_actual_y_tendencias_de_la _radiotelevision_publica_en_Europa

Chalmers, D., Danson, M., Lang, A. y Milligan, L. (2012). The Contribution of BBC ALBA to Gaelic: A Social and Economic Review. Social Media and minority languages: Convergence and the creative industries, 152, 212.

Cormack, M. y Hourigan, N. (Eds.). (2007). Minority Language Media: Concepts, Critiques and Case Studies. Clevedon (UK): Multilingual Matters.

Croteau, D. y Hoynes, W. (2001). The business of media: Corporate media and the public interest. Londres: Pine Forge Press-Sage. 
Cunliffe, D., Pearson, N. y Richards, S. (2010). E-commerce and Minority languages: a Welsh perspective. En H. Kelly-Holmes y G. Mautner (Eds.), Language and the Market (pp. 135-147): Palgrave Macmillan.

Doyle, G. (2002). Understanding Media Economics. Londres: Sage.

Dragomir, M. (2018). Control the money, control the media: How government uses funding to keep media in line. Journalism, 19(8), 1131-1148.

https://doi.org/10.1177\%2F1464884917724621

Elliott, L. (2011, 7 de agosto). Global financial crisis: five key stages 2007-2011. The Guardian. Recuperado de https://www.theguardian.com/business/2011/aug/07/global-financial-crisis-keystages

Ferré-Pavia, C., Zabaleta, I., Gutierrez, A., Fernandez-Astobiza, I. y Xamardo, N. (2018). Internet and Social Media in European Minority Languages: Analysis of the Digitalization Process. International Journal of Communication, 12, 1065-1086. Recuperado de https://ijoc.org/index.php/ijoc/article/view/7464

Franklin, B. (Ed.) (2016). The Future of Journalism: In an age of digital media and economic uncertainty. Oxford (UK): Taylor \& Francis.

Gorter, D., Gorter, D., Hoekstra, J., Jansma, L. G. y Ytsma, J. (Eds.). (1989). Fourth International COnference on Minority Languages, Vol. 1: General Papers. Clevedon (UK): Multilingual Matters.

Grin, F., Sfreddo, C. y Vaillancourt, F. (2010). The Economics of the Multilingual Workplace. London: Routledge.

Grin, F. y Vaillancourt, F. (1999). The cost-effectiveness evaluation of minority language policies: Case studies on Wales, Ireland and the Basque Country (Vol. 2). Flensburg (Germany): European Centre for Minority Issues (ECMI). 
Hanley, R. (2003). Death of public broadcasting?: New funding model needed to stave off NPR, PBS extintion. Recuperado de www.msnbc.com/news/925728.asp?cp1=1\#BODY

Hogan-Brun, G. y O'Rourke, B. (2018). The Palgrave Handbook of Minority Languages and Communities: Palgrave Macmillan UK.

Hogan-Brun, G. y Wolff, S. (Eds.). (2003). Minority languages in Europe: Frameworks, Status, Prospects. London: Palgrave MacMillan.

Jones, E. H. G. y Uribe-Jongbloed, E. (Eds.). (2012). Social Media and Minority Languages: Convergence and the Creative Industries. Bristol (UK): Multilingual Matters. Kevin, D., Pellicanò, F. y Schneeberger, A. (2013). Television News Channels in Europe. Obtenido de https://rm.coe.int/16807835e1

Kirk, J. y Baoill, D. P. Ó. (2003). Towards our Goals in Broadcasting, the Press, the Performing Arts and the Economy: Minority Languages in Northern Ireland, the Republic of Ireland, and Scotland. Belfast: Queens's University Belfast.

LEAP/Europe 2020, L. E. d. A. P. (2011). GEAB N59 - Contenidos. GEAB, GlobalEurope Anticipation Bulletin, 2011(17-11-2011). Recuperado de www.leap2020.eu/GEAB-N59-Contenidos_a2919.html

Levy, D. A. L. y Nielsen, R. K. (Eds.). (2010). The Changing Business of Journalism and its Implications for Democracy. Oxford (UK): Reuters Institute for the Study of Journalism, University of Oxford.

Lowe, G. F. y Berg, C. E. (2013). The Funding of Public Service Media: A Matter of Value and Values. International Journal on Media Management, 15(2), 77-97.

https://doi.org/10.1080/14241277.2012.748663

McQuail, D. (2000/1983). McQuail's Mass Communication Theory (4a edición. Reimpreso en 2002). Londres: Sage. 
MG Alba. (2020). Annual Report \& Statement of Accounts 19/20. Recuperado de www.mgalba.com/downloads/reportsf/annual-report-19-20.pdf?lang=en

Moring, T. (2007). Functional Completeness in Minority Language Media. En M. Cormack y N. Hourigan (Eds.), Minority Language Media: Concepts, Critiques and Case Studies (pp. 17-33). Clevedon (UK): Multilingual Matters.

Mylonas, Y. (2012). Media and the Economic Crisis of the EU: The 'Culturalization' of a Systemic Crisis and Bild Zeitung's Framing of Greece. Triple C: Cognition, Communication, Co-operation, 10(2), 646-671. Recuperado de http://www.triple-c.at

Nobre Correia, J. M. (2010). La Europa de los medios frente a la crisis IRC, Infoamérica, (2), 89-104. Recuperado de www.infoamerica.org/icr/n02/nobre\%20_correia.pdf

Pietikäinen, S., Kelly-Holmes, H. y Rieder, M. (2019). Minority Languages and Markets. En G. Hogan-Brun y B. O'Rourke (Eds.), The Palgrave Handbook of Minority Languages and Communities (pp. 287-310). London: Palgrave Macmillan UK.

Riggins, S. H. (Ed.) (1992). Ethnic minority media : an international perspective. Newbury Park, Calif.: Sage Publications.

Rogers, E. (1995). The difussion of innovations. New York: Free Press.

S4C. (2020). Annual Report and Statement of Accounts for the 12 month period to 31 March 2020. Recuperado de https://dlo6cycw1kmbs.cloudfront.net/media/media_assets/s4c-adroddiad-blyn2019-20.pdf

Tunstall, J. (1991). A media industry perspective. ENJ. Anderson (Ed.), Communication Yearbook 14 (pp. 163-186). Newbury Park, CA: Sage.

Westcott, T. (2015). The Future of Public Broadcasting: Funding Strategies From Around the World. Recuperado de http://www.miptrends.com/wpcontent/uploads/2016/03/miptv-mipcom-ihs-public-broadcasting-white-paper.pdf 
Iñaki Zabaleta Urkiola, Tania Arriaga Azkarate, María Gorosarri González y Nicolás Xamadro González

Wimmer, R. D. y Dominick, J. R. (2000). La investigación científica de los medios de comunicación. Barcelona: Bosch Comunicación.

Zabaleta, I., Ferre-Pavia, C., Gutiérrez, A., Fernández, I. y Xamardo, N. (2014). European minority language media and journalism: Framing their marginal reality. International Communication Gazette, 76(3), 275-295.

https://doi.org/10.1177\%2F1748048513516907

Zabaleta, I., Gutierrez, A., Ferré Pavia, C., Fernandez, I., Urrutia, S. y Xamardo, N. (2013). Website Development and Digital Skill: The State of Traditional Media in European Minority Languages. International Journal of Communication, 7(2013), 16411666. Recuperado de https://ijoc.org/index.php/ijoc/article/view/1986

Zabaleta, I., Gutierrez, A., Ferré-Pavia, C., Fernandez, I. y Xamardo, N. (2019). Facts and transformations in European minority language media systems amid digitalization and economic crisis. International Communication Gazette, 81(3), 235-258.

https://doi.org/10.1177\%2F1748048518754749

Zabaleta, I. y Xamardo, N. (2020). Economy and funding of European minority language media: Reality and impact of digitalization and economic crisis. Journalism (online). https://doi.org/10.1177\%2F1464884920954039

Zabaleta, I., Xamardo, N., Gutierrez, A., Urrutia, S., Fernandez, I. y Ferré, C. (2010). Beween Language Support and Activism. Journalism Studies, 11(2), 190-208. https://doi.org/10.1080/14616700903290668

\footnotetext{
'Emplearemos ambos términos para significar que la "minorización" de una comunidad lingüística es un proceso complejo y normalmente largo en el tiempo, en el que siempre hay un cierto grado de coerción externa y abandono interno.

ii Véase nota de prensa- Comisión Europea: ' 10 years since the start of the crisis: back to recovery thanks to decisive EU action'. Publicada el 9/8/2017 (Consultado el 18/6/18). URL: http://europa.eu/rapid/press-release_IP-17-2401 en.htm

iii Estos datos estadísticos deben tomarse como estimaciones aproximadas, ya que existen diferentes tipos y gradaciones de competencia lingüística, así como rangos de edad. Las fuentes consultadas son agencias de estadística oficiales: Oficina de Gales para Estadísticas Nacionales (ONS; www.ons.gov.uk/census); La Oficina Central de Estadísticas de Irlanda (http://www.cso.ie) y la Agencia de Investigación y Estadísticas de Irlanda del Norte (NISRA; /www.nisra.gov.uk); Registros nacionales escoceses-gaélicos de
} 
Escocia (Censo de Escocia; www.scotlandscensus.gov.uk). En el caso irlandés, el número total de hablantes de irlandés, incluidos el norte y el sur, según el censo de 2011, asciende a 774.893, pero esta cifra ha sido cuestionada por algunas fuentes irlandesas que trabajan en medios irlandeses.

iv Y Cymro, el histórico periódico semanal de información y difusión generales, se cerró en 2017.

$\checkmark$ URL de la carta: http://conventions.coe.int/Treaty/Commun/ChercheSig.asp?NT=148\&CM=\&DF=\&CL=ENG) . URL de la Convención: http://www.co'e.int/en/web/minorities/home.

vi Los viajes a Irlanda y Gales se realizaron en 2016, 2017 y 2019. En el caso de Escocia la información se recogió por correo electrónico y mediante conversaciones telefónicas durante dichos años.

vii Una de las preguntas fue: “¿Ha afectado a su medio de comunicación la crisis económica que se expandió en Europa después de 2008? Por favor, explíquelo". La segunda pregunta se redactó del siguiente modo: “¿Cuál es su opinión sobre la financiación y la ayuda pública que reciben? ¿Es suficiente? Por favor, explíquelo".

viii Esta plataforma digital de la BBC es codificada como cibermedio autónomo porque Radio Cymru, la radio en galés de la BBC, tiene su propio sitio web (www.bbc.co.uk/radiocymru), dedicado a servir de plataforma de la programación de la radio.

ix Véase BBC Full Financial Statements, https://downloads.bbc.co.uk/aboutthebbc/insidethebbc/reports/pdf/BBC-FS-2016.pdf. En Irlanda, RTÉ: :https://static.rasset.ie/documents/about/rte-annual-report-2015.pdf. 